nodosum leprosum and lupus profundus panniculitis, immunologic mediated conditions responding well to thalidomide. There has been so far only one other report of thalidomide use in WCD. 24 years ago a favourable response in a 23 y.o. Female from Malaysia was reported. This patient had a remission, which was ongoing despite stopping of all medications after treatment with thalidomide of only 13 weeks. The question of a possible spontaneous remission asked by the describing authors remains unanswered. To the contrary, in the here described case repeated reduction of thalidomide below $100 \mathrm{mg} / \mathrm{dl}$ caused mild recurrence of sympt., responding immediately to dose increase to 100 mg.

Thalidomide?s anti-TNFa modulatory and anti-angiogenic effects have recently been rediscovered also for treatment of other autoimmune diseases. Superior tolerability and side-effect profile (compared to corticosteroids and immunosuppressives) make thalidomide a first line treatment consideration for chronic WCD in patients without childbearing potential.

\section{THU0219 RETROSPECTIVE EVALUATION OF 180 PATIENTS WHO WERE DIAGNOSED AS UNDIFFERENTIATED CONNECTIVE TISSUE DISEASE}

E Doganavsargil, F Oksel, G Keser, V Inal, K Aksu. Rheumatology, Ege University Hospital, Izmir, Turkey

\subsection{6/annrheumdis-2001.751}

\section{Background}

Objectives To review the outcome and diagnosis of patients whose initial clinical and laboratory findings were suggestive of a well established and undifferentiated connective tissue disease (uCTD), without fulfilling the diagnostic criteria of any connective tissue disease.

Methods Medical records of patients being followed up by Ege University rheumatology department between 1990 and 1999 were retrospectively screened. The data of 478 patients, constituting $18 \%$ of the total, whose initial diagnosis was uCTD, were further investigated. Patients with missing data were omitted and only 180 patients having reliable and complete initial clinical and laboratory data with regular controls were included.

Results The mean ages of 180 patients (F/M:173/7) at the time of initial clinical symptoms and at the time of diagnosis of uCTD were $35 \pm 12$ years and $38 \pm 12$ years, respectively. The distribution of initial symptoms and clinical findings were as follows: arthralgia/arthritis 97.2\%, Raynaud phenomenon (RP) $30.5 \%$, mucocutaneous findings $68.2 \%$, symptoms of keratoconjunktivitis sicca $14.4 \%$. It was notable that none of the patients had presented with initial symptoms or clinical finding reflecting direct involvement of a specific organ.

In the final evaluation, 46 out of 180 patients (25.5\%) were found to evolve into a definite clinical diagnosis: Sjögren's syndrome 21 (11.6\%), systemic lupus erythematosus $8(4.4 \%)$, rheumatoid arthritis 8 (4.4\%), CREST syndrome 7 (3.8\%), diffuse systemic sclerosis 1 and seronagative polyarthritis 1 . In 5 patients a nonrheumatologic diagnosis was made, while in 11 patients fibromyalgia was diagnosed. The diagnosis of 118 $(65 \%)$ patients (F/M:115/3) remained unchanged.

The evaluation of initial clinical and laboratory parameters with respect to their contribution to final diagnosis revealed that, presence of Raynaud phenomenon and anti-centromeric antibodies were highly suggestive of later diagnosis of limited ScS. On the other hand, anti-dsDNA antibodies, leucopenia and thrombocytopenia, which are important parameters in well established SLE, were initially unpredictive of later SLE development in patients whose initial diagnosis was uCTD.

Conclusion In most of the patients whose initial diagnosis was $\mathrm{uCTD}$, the disease tends to remain undifferentiated.

\section{THU0220 AN ESTIMATION OF THE PREVALENCE OF HYPERMOBILITY}

${ }^{1} \mathrm{M}$ Gumà, ${ }^{2} \mathrm{~J}$ Roca, ${ }^{1} \mathrm{~S}$ Holgado, 'M Valls-Roc, ${ }^{1} \mathrm{E}$ Casado, ${ }^{3} \mathrm{~J}$ Forcada, ${ }^{1} \mathrm{JC}$ Duro, ${ }^{1} \mathrm{~A}$ Olive, ${ }^{1} \mathrm{X}$ Tena. ${ }^{1}$ Rheumatology; ${ }^{2}$ Epidemiology; ${ }^{3} G$ P, Hospital Universitari Germans Trias I Pujol, Badalona, Spain

\subsection{6/annrheumdis-2001.752}

Background Hypermobility is associated with several rheumatic manifestations. The association with SLE is controversial.

Objectives To determine the prevalence of hypermobility in two different populations: a general practitioner (GP) office and outpatients clinic of a referral rheumatology hospital.

Methods 280 patients (193 females, 87 males); mean age 44.7 \pm 11.2 were consecutively reviewed in a GP office. 370 patients (233 females, 84 males); mean age $50.5 \pm 16.8$ were consecutively visited at the out-patients rheumatology clinic. Hypermobility was evaluated following the criteria of Carter-Beighton (hypermobility $>4$ points).

Results Hypermobility was present in $42 / 280$ (15\%) at the GP office and in $81 / 317(25 \%)$ in the out patients rheumatology clinic.

\begin{tabular}{lllll}
\multicolumn{5}{l}{ Abstract THU0220 Table 1} \\
\hline General practitioner & \multicolumn{3}{l}{ Hospital } \\
\hline Hypermobility & YES & NO & YES & NO \\
Age & $28 \pm 11.7$ & $47 \pm 18.3$ & $38.9 \pm 14.2$ & $54 \pm 15.9$ \\
Sex (F/M) & $5.8 / 1$ & $1 / 1.2$ & $3,7 / 1$ & $1 / 1.3$ \\
Criteria & $5.1 \pm 1$ & $0.6 \pm 1$ & $5.6 \pm 1.6$ & $0.5 \pm 0.9$ \\
\hline
\end{tabular}

Conclusion The prevalence of hypermobility at the GP office and a rheumatology clinic is high. Furthermore it is close to the prevalence of hypermobility in the general population.

\section{THU0221 OSTEONECROSIS AND HIV INFECTION: REPORT OF ELEVEN CASES}

${ }^{1} \mathrm{M}$ Valls-Roc, ${ }^{1} \mathrm{~V}$ Ortiz-Santamaria, ${ }^{1} \mathrm{~A}$ Olive, ${ }^{2} \mathrm{M}$ Cervantes, ${ }^{2} \mathrm{G}$ Gratacos, ${ }^{3} \mathrm{G}$ Sirera, ${ }^{3} \mathrm{C}$ Tural, ${ }^{3}$ E Negredo, ${ }^{1} \mathrm{X}$ Tena, ${ }^{3} \mathrm{~B}$ Clotet. ${ }^{1}$ Rheumatology Service, Hospital Universitari Germans Trias I Pujol, Badalona, Spain; ${ }^{2}$ Rheumatology Service, Consorci Sanitari Parc Tauli, Sabadell, Spain; ${ }^{3}$ HIV Unit, Hospital Universitari Germans Trias I Pujol, Badalona, Spain

\subsection{6/annrheumdis-2001.753}

Background A vascular necrosi (AVN) has been described in the setting of HIV infection. No epidemiological studies to determine the higher incidence of AVN in HIV+ patients are available. Protease inhibitor containing regimens (PI-CR) have been considered an additional risk factor for developing AVN.

Objectives To report the clinical findings of $11 \mathrm{HIV}+$ patients with AVN.

Methods Retrospective review of clinical and radiological charts of 11 patients diagnosed of AVN. 